\title{
Ex Vivo Assessment of Coronary Atherosclerotic Plaque by Grating-Based Phase-Contrast Computed Tomography Correlation With Optical Coherence Tomography
}

\author{
Christopher Habbel, MD, * Holger Hetterich, MD, *† Marian Willner, PhD, $\neq$ Julia Herzen, PhD, $\neq$ \\ Kristin Steigerwald, Dr vet med, $\S$ Sigrid Auweter, PhD, * Ulrich Schüller, MD,//q\#** Jörg Hausleiter, MD, $\dagger+$ \\ Steffen Massberg, MD, $+\neq \neq$ Maximilian Reiser, MD, * Franz Pfeiffer, PhD, $\neq$ \\ Tobias Saam, MD, * and Fabian Bamberg, $M D^{*} \xi \xi$
}

\begin{abstract}
Objectives: The aim of this study was to determine the diagnostic accuracy of grating-based phase-contrast computed tomography (gb-PCCT) to classify and quantify coronary vessel characteristics in comparison with optical coherence tomography (OCT) and histopathology in an ex vivo setting.

Materials and Methods: After excision from 5 heart specimens, 15 human coronary arteries underwent gb-PCCT examination using an experimental imaging setup consisting of a rotating molybdenum anode $\mathrm{x}$-ray tube, a Talbot-Lau grating interferometer, and a single photon counting detector. Subsequently, all vessels were imaged by OCT and histopathologically processed. Optical coherence tomography, gb-PCCT, and histopathology images were manually matched using anatomical landmarks. Optical coherence tomography and gb-PCCT were reviewed by 2 independent observers blinded to histopathology. Vessel, lumen, and plaque area were measured, and plaque characteristics (lipid rich, calcified, and fibrous) were determined for each section. Measures of diagnostic accuracy were derived, applying histopathology as the standard of reference.

Results: Of a total of 286 assessed cross sections, 241 corresponding sections were included in the statistical analysis. Quantitative measures derived from gb-PCCT were significantly higher than from OCT $(P<0.001)$ and were strongly correlated with histopathology (Pearson $r \geq 0.85$ for gb-PCCT and $\geq 0.61$ for OCT, respectively). Results of Bland-Altman analysis demonstrated smaller mean differences between OCT and histopathology than for gb-PCCT and histopathology. Limits of agreement were narrower for gb-PCCT with regard to lumen area, for OCT with regard to plaque area, and were comparable with regard to vessel area. Based on histopathology, 228/241 (94.6\%) sections were classified as fibrous, calcified, or lipid rich. The diagnostic accuracy of gb-PCCT was excellent for the detection of all plaque components (sensitivity, $\geq 0.95$; specificity, $\geq 0.94$ ), whereas the results for OCT showed sensitivities of $\geq 0.73$ and specificities of $\geq 0.66$.
\end{abstract}

Received for publication August 1, 2016; and accepted for publication, after revision, November 10, 2016

From the *Institute of Clinical Radiology, Ludwig-Maximilian University; $\dagger$ German Center for Cardiovascular Disease Research (DZHK e.V.), Munich; $₫$ Lehrstuhl für Biomedizinische Physik, Physik-Department \& Institut für Medizintechnik, Technische Universität München, Garching; §German Heart Center of the State of Bavaria and the Technical University Munich; \|Center for Neuropathology, Ludwig-Maximilian University, Munich; 9 Institute of Neuropathology, University Medical Center Hamburg-Eppendorf; \#Research Institute Children's Cancer Center; **Department of Pediatric Hematology and Oncology, University Medical Center Hamburg-Eppendorf, Hamburg; $\dagger \dagger$ Institute for Medical Information Sciences, Biometry and Epidemiology, Ludwig-Maximilian University, Munich; $\ddagger$ Medizinische Klinik I and Poliklinik, Ludwig-Maximilian University, Munich; and $\S$ Department of Diagnostic and Interventional Radiology, University of Tübingen, Tübingen, Germany.

Christopher Habbel and Holger Hetterich contributed equally to this work.

Conflicts of interest and sources of funding: none declared.

Supplemental digital contents are available for this article. Direct URL citations appear in the printed text and are provided in the HTML and PDF versions of this article on the journal's Web site (www.investigativeradiology.com).

Correspondence to: Holger Hetterich, MD, Institute of Clinical Radiology, LudwigMaximilians-University Hospital, Marchioninistraße 15, 81377 Munich, Germany. E-mail: Holger.Hetterich@med.uni-muenchen.de.

Copyright $\mathbb{C} 2017$ Wolters Kluwer Health, Inc. All rights reserved.

ISSN: 0020-9996/17/5204-0223

DOI: $10.1097 /$ RLI.0000000000000346
Conclusions: In this ex vivo setting, gb-PCCT provides excellent results in the assessment of coronary atherosclerotic plaque characteristics and vessel dimensions in comparison to OCT and histopathology. Thus, the technique may serve as adjunct nondestructive modality for advanced plaque characterization in an experimental setting.

Key Words: x-ray phase-contrast computed tomography, coronary arteries, optical coherence tomography, atherosclerosis

(Invest Radiol 2017;52: 223-231)

A dvanced imaging techniques have contributed greatly to our current understanding of the pathophysiology of atherosclerotic disease initiation and progression. They play a pivotal role in numerous studies including both experimental and clinical research. ${ }^{1,2}$ Intravascular imaging techniques such as intravascular ultrasound and optical coherence tomography (OCT) offer excellent soft tissue contrast and spatial resolution, thus allowing for detailed assessment of atherosclerotic lesions. However, also in an ex vivo setting, they are limited with regard to penetration depth and 3-dimensional (3D) assessment of vessel anatomy. ${ }^{3}$ Thus a noninvasive imaging method for accurate plaque characterization is desirable. Currently, computed tomography (CT) and magnetic resonance imaging are the methods of choice to characterize atherosclerotic plaques noninvasively, although both suffer from certain limitations. Coronary CT angiography allows fast and robust data acquisition and provides excellent insight into the gross vessel anatomy, ${ }^{4}$ such as vessel lumen or degree of vessel stenosis. The degree of calcification as assessed by cardiac CT provides information on the overall extent of coronary atherosclerosis and valuable prognostic information. ${ }^{5}$ However, coronary CT angiography offers only few details on soft plaque composition, because noncalcified plaque components, including fibrous and fatty tissue, display substantial overlap in their density profiles and are thus difficult to differentiate. ${ }^{2,6}$

Phase-contrast CT is an $\mathrm{x}$-ray-based imaging technique providing high contrast, including low absorbing materials, such as biological soft tissue. $^{7,8}$ Unlike other more common $\mathrm{x}$-ray imaging techniques, the technique does not rely on $\mathrm{x}$-ray attenuation, but on $\mathrm{x}$-ray phase shift. ${ }^{9,10}$ Studies have shown that the image contrast of different tissue entities can be several magnitudes higher in phase-contrast- than in absorptioncontrast-based imaging techniques. ${ }^{2,6,11}$ Its major limitation was its dependency on monochromatic x-ray sources such as synchrotron radiation, hampering the widespread use of this technology. Recently, introduced grating-based phase-contrast imaging allows for the use of polychromatic x-ray sources such as conventional x-ray tubes. ${ }^{9,12}$ Earlier studies have demonstrated the potential of grating-based phase-contrast CT (gb-PCCT) for atherosclerotic plaque assessment using histopathology as standard of reference. ${ }^{2}$ However, the performance of gb-PCCT in comparison to an established advanced high-resolution imaging modality, such as OCT, is unknown.

Therefore, the aim of this study was to evaluate the feasibility of gb-PCCT for the comprehensive assessment of coronary artery 
atherosclerotic plaque in comparison to OCT using histopathology as the standard of reference. We hypothesized that gb-PCCT provides nondestructive assessment of vessel dimensions and plaque composition similar to OCT.

\section{MATERIALS AND METHODS}

\section{Study Design and Experimental Setup}

The present study was designed as a postmortem, ex vivo experimental study. The project was approved by the local ethics committee and complies with the Declaration of Helsinki of 1975, as revised in 2008. Heart specimens were provided by the International Institute for Advancement in Medicine (IIAM, Edison, NJ). Written informed consent to grant tissue for research purposes were obtained by the donor or the donor's relatives.

Heart specimens were obtained within 24 hours postmortem. The coronary artery specimens (right coronary artery [RCA], left main coronary artery [LM], left anterior descending coronary artery [LAD], and left circumflex coronary artery [LCX]) including parts of the epicardial fat and myocardium were excised and stored in neutral buffered formalin solution $(10 \%)$. Specimens were first imaged by OCT as well as gb-PCCT and underwent histopathology workup. Histopathology, OCT and gb-PCCT data sets were matched using multiple anatomical landmarks. Lumen, vessel, and plaque areas were measured in histopathology, OCT, and gb-PCCT independently. Measures of diagnostic accuracy for the detection of calcified, fibrous, and fatty tissue were obtained for OCT and gb-PCCT using histopathology as the standard of reference.

\section{Gb-PCCT Experimental Setup and Data Processing}

The gb-PCCT setup described in detail in Birnbacher et $\mathrm{al}^{13}$ consisted of an $\mathrm{x}$-ray tube (ENRAF Nonius rotating molybdenum anode $\mathrm{x}$-ray tube) operating at a voltage of $40 \mathrm{kV}$ and a tube current of $70 \mathrm{~mA}$. The optical transmission gratings with periods of $5.4 \mu \mathrm{m}$ were produced by Microworks (Karlsruhe, Germany). A gold absorption grating with a height of $50 \mu \mathrm{m}$ was positioned directly behind the $\mathrm{x}$-ray source. This grating increases coherence of the $\mathrm{x}$-rays produced by an incoherent $x$-ray source. During the penetration of the specimen, the x-rays experience a phase shift and hit the nickel phase grating (height, $8 \mu \mathrm{m}$ ), which creates an interference pattern in a certain distance according to the Talbot effect. This interference pattern carries information about the structure of the specimen. The analysis of this pattern requires a third gold analyzer grating (height, $50 \mu \mathrm{m}$ ), which translates the interference pattern into intensity modulations detectable by an x-ray detector (single photon counting detector Pilatus II, Dectris, Baden, Switzerland, $487 \times 195$ pixels, $172 \times 172 \mu \mathrm{m}^{2}$ pixel size). With an intergrating distance of $87.5 \mathrm{~cm}$, this setup results in a 1.72-fold magnification and an effective pixel size of $100 \times 100 \mu \mathrm{m}^{2}$. A number of 1200 projections over 360 degrees were recorded for a full tomographic scan ${ }^{12,14}$ For every 20 projections with specimen, 5 reference projections without specimen were obtained. The phase grating was stepped over 1 period for each projection and reference projection while 11 images were acquired. Exposure time per projection was 5 seconds. For the reconstruction of the phase-contrast data, the standard filtered backprojection algorithm with an imaginary Hilbert filter was used. Data were stored in DICOM format.

\section{OCT Experimental Setup}

Optical coherence tomography examinations were performed using an ILUMIEN PCI Optimization System (St Jude Medical Inc, St Paul, MN) and a Dragonfly Duo OCT Imaging Catheter. ${ }^{15}$ The specimens were flushed with normal saline to clean the vessel of any remaining blood clots. Afterward, the imaging catheter was introduced as far distally into the vessel as possible. Images were acquired during an automated pullback (pullback speed, $20 \mathrm{~mm} / \mathrm{s}$ ) over a maximal length of $50 \mathrm{~mm} .{ }^{15}$ If necessary, several pullbacks were performed to cover the full length of the vessel. External sutures marked the positions of the catheter at the beginning and at the end of each pullback. Images were stored in DICOM format.

\section{Histopathology Workup and Analysis}

After completion of all imaging experiments, vessel specimens were decalcified, dehydrated, and embedded in paraffin. Serial sections were taken. Thickness of the sections was $10 \mu \mathrm{m}$, taken every $1 \mathrm{~mm}$. Staining was performed with hematoxylin-eosin. Images were scanned using a dedicated camera (AVT Stingray F-146 Fire Wire CCD, pixel resolution $0.23 \mu \mathrm{m}$; Carl Zeiss, Jena, Germany). The lumen and vessel borders were manually traced in all cross sections. Atherosclerosis was defined as the presence of either fibrous (FIB), lipid rich (LIP), or calcified tissue (CAL) on histopathology. The presence or absence of each plaque component on a cross-sectional basis was evaluated by a pathologist with more than 10 years of experience in vascular pathology who was blinded to the gb-PCCT and OCT data sets.

\section{Gb-PCCT, OCT, and Histopathology Matching}

Optical coherence tomography images, gb-PCCT, and histopathology data were manually matched by an independent investigator, who was not involved in further analysis, using multiple anatomical landmarks, such as plaque formation, vessel shape and lumen size, bifurcations, as well as relative distance to side branches and smaller vessels ${ }^{16}$ (see Supplement 1 , Supplemental Digital Content 1, http://links.lww.com/RLI/A305).

\section{Analysis of Lumen, Vessel, and Plaque Area}

Grating-based phase-contrast CT and OCT data were evaluated several weeks apart by 2 independent observers with more than 5 years of experience in cardiovascular imaging and substantial expertise in gbPCCT and OCT blinded to the histopathology data set. Previously published signal criteria were used to identify the lumen and vessel border in gb-PCCT. ${ }^{2,6,17}$ The lumen and vessel borders were manually traced in all corresponding sections for both gb-PCCT and OCT measurements. Details on how measurements were performed can be found in Supplement 1, Supplemental Digital Content 1, http://links.lww.com/ RLI/A305. Plaque area was calculated by subtracting lumen area from vessel area for both gb-PCCT and OCT analysis., ${ }^{2,6,17}$

\section{Plaque Detection and Differentiation}

Two experienced observers blinded to the histopathology data analyzed and classified atherosclerotic plaque and its components. Using previously found criteria, ${ }^{2,6,17}$ the presence or absence of atherosclerosis and the presence or absence of FIB, LIP, and CAL was assessed independently by both readers. In case of disagreement, a consensus between the 2 readers was reached by conjoint reading and discussion

\section{Statistical Data Analysis}

Statistical analyses were performed using "Statistical Package for the Social Sciences" (SPSS) Version 23 software (IBM, Armonk, NY). Measurements of diagnostic accuracy, namely, specificity, sensitivity, negative predictive value (NPV), and positive predictive value (PPV) including 95\% confidence intervals (CIs), were calculated. Furthermore, Cohen $\kappa$ was calculated to assess the agreement of gb-PCCT and OCT with histopathology. Normal distributed numerical results are shown as mean \pm standard deviation (SD). For quantitative measures of lumen, vessel, and plaque area, the mean \pm SD was calculated for gbPCCT and OCT data, as well as for histopathology data. The Wilcoxon test was used for the comparison between gb-PCCT and OCT. A $P$ value of less than 0.05 was used to indicate statistical significance. To evaluate the correlation for gb-PCCT and OCT with histopathology, Pearson 
correlation coefficient $r$ was calculated and Bland-Altman plot analyses were performed.

\section{RESULTS}

Initially, 286 corresponding gb-PCCT, OCT, and histopathology cross sections were derived from 15 arteries $(n=5$ RCA, $n=5$ LM/ $\mathrm{LAD}, \mathrm{n}=5 \mathrm{LCX})$. In OCT, 25/286 (8.7\%) sections were excluded, $19 / 286(6.6 \%)$ due to artifacts and 6/286 (2.1\%) due to large vessel diameter, respectively. In gb-PCCT, 9/286 (3.1\%) sections were excluded, $5 / 286(1.7 \%)$ due to extensive calcifications and $4 / 286(1.4 \%)$ due to air bubbles within the vessel. In histopathology, 11/286 (3.8\%) slices showed substantial artifacts and were not included in the analysis. Thus, a total number of 241/286 (84.3\%) corresponding gb-PCCT, OCT, and histopathology cross sections were available for analysis (Fig. 1). Figure 2 shows examples of different plaques in gb-PCCT, OCT, and histopathology containing different components such as FIB, LIP, and CAL. Figure 3 shows a longitudinal reconstruction of an LAD and its corresponding cross sections in gb-PCCT and OCT as well as histopathology.

\section{Analysis of Lumen, Vessel, and Plaque Area}

Mean values of lumen, vessel, and plaque areas in gb-PCCT, OCT, and histopathology measurements are summarized in Tables 1 and 2. In comparison to histopathology, both gb-PCCT and OCT were associated with significantly higher values for lumen, vessel, and plaque area $(P<0.0001)$. Furthermore, gb-PCCT measurements provided higher values for the lumen, vessel, and plaque area than OCT $(P<0.0001)$. There was a strong correlation between gb-PCCT and histopathology $(r=0.90, r=0.77$, and $r=0.89$ for lumen, vessel, and plaque area, respectively, all $P<0.0001)$ and good correlation between OCT and histopathology $(r=0.64, r=0.65$, and $r=0.61$, respectively, all $P<0.0001)$. Bland-Altman plots, indicating mean differences and limits of agreement for measurements of lumen, plaque, and vessel dimensions, for the correlation of gb-PCCT and histopathology as well as OCT and histopathology are shown in Figures 4 and 5, respectively. In short, mean differences between OCT and histopathology were smaller than for gb-PCCT and histopathology. Limits of agreement were narrower for gb-PCCT with regard to lumen area, for OCT with regard to plaque area, and were comparable with regard to vessel area.

\section{Plaque Detection and Differentiation}

According to histopathology, the prevalence of any atherosclerotic plaque was 228/241 (94.6\%). Plaque components were detected as follows: FIB, 228/241 (94.6\%); LIP, 74/241 (30.7\%); and CAL,

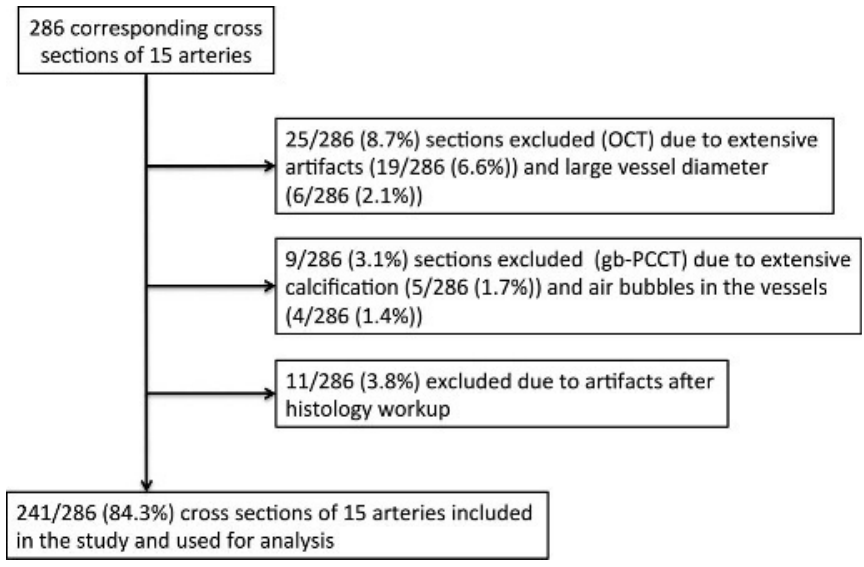

FIGURE 1. Exclusion of cross sections. A total number of 241/286 (84.3\%) corresponding cross sections were included in the study and used for analysis.
135/241 (56.0\%). For gb-PCCT, sensitivities, specificities, PPVs, and NPVs ranged between 0.88 and 1.00 for all plaque tissues $(P<0.0001)$ with $\kappa$-values of 0.96 for FIB, 0.97 for CAL, and 0.89 for LIP. For OCT, sensitivities, specificities, PPVs, and NPVs ranged between 0.60 and $1.00(P<0.0001)$. Diagnostic accuracy was lowest for the detection of LIP with a sensitivity of 0.80 , specificity of 0.69 , PPV of 0.60 , and NPV of $0.85(P<0.0001)$; $\kappa$-values were 0.81 for FIB, 0.50 for LIP, and 0.62 for CAL. Details on diagnostic accuracy estimates are provided in Tables 3 and 4, respectively.

\section{DISCUSSION}

The results of the present study indicate that gb-PCCT is an accurate tool to comprehensively assess coronary atherosclerotic plaque in comparison with histopathology in an ex vivo setup. In this setting, gb-PCCT showed higher diagnostic accuracy than OCT for the detection and differentiation of important atherosclerotic plaque components, including fibrous, lipid-rich, and calcified tissue. Furthermore, gb-PCCT allowed an accurate quantification of vessel, plaque, and lumen dimensions with better correlation to histopathology than OCT.

Grating-based phase-contrast imaging has recently been introduced as a novel $x$-ray-based imaging method that provides additional value in several areas, including pulmonary ${ }^{18-25}$ and breast imaging. ${ }^{26-34}$ In addition, initial evidence suggests that gb-PCCT is able to detect and characterize atherosclerotic plaque lesions in coronary and carotid arteries. ${ }^{6,35}$ Previous studies were able to show the superior diagnostic accuracy of gb-PCCT in comparison to conventional absorption $\mathrm{CT}$, for the quantitative and qualitative characterization of atherosclerotic plaques using a high-resolution synchrotron setup. ${ }^{36}$ In contrast, gb-PCCT was performed with a conventional $\mathrm{x}$-ray source in the present study. The use of a conventional $\mathrm{x}$-ray source is associated with a reduced resolution as well as reduced signal-to-noise ratio. ${ }^{16}$ However, it has been shown that critical structures of atherosclerotic plaque can be differentiated using either X-ray tubes or synchrotron radiation. ${ }^{2,10,36}$ Furthermore, the use of a conventional x-ray tube for gb-PCCT may facilitate the widespread use of this technology due to its independence of synchrotron facilities with limited access and capacities and is also closer to potential clinical applications. Optical coherence tomography is an established, accurate imaging tool for the analysis of quantitative vessel parameters, vessel anatomy, and atherosclerotic plaque lesions. 3,37-39 However, the performance of gb-PCCT in a headto-head comparison to an established advanced high-resolution imaging modality, such as OCT, was unknown so far.

Our data show significant absolute differences relative to histopathology for the quantitative assessment of lumen, vessel, and plaque area for both OCT and gb-PCCT. Average discrepancies were lower for OCT versus histopathology than gb-PCCT versus histopathology. Most likely, these discrepancies may be explained by tissue shrinkage during histopathological workup, which has been documented previously ${ }^{6}$ and vessel configuration during the imaging procedure. Furthermore, observed differences between OCT and gb-PCCT may be attributable to differences in the tilting angle during measurements, limited penetration depth of OCT that may not have optimally visualized the complete vessel wall in larger vessels, and outside compression of the vessel during the OCT or gb-PCCT imaging process. Thus a comparison of absolute values, absolute differences to histopathology, and also the limits of agreement in the Bland-Altman analysis have to be regarded and interpreted with caution. However, despite higher differences in absolute numbers, we observed a better correlation of gb-PCCT with histopathology than OCT with histopathology for lumen, vessel, and plaque underlining the potential for precise quantitative vessel assessment. Furthermore, gb-PCCT yields complete 3D data sets that allow multiplanar reformatting (see Fig. 3). Overall, our results suggest that in an ex vivo setting, gb-PCCT measures of vessel, lumen, and plaque 


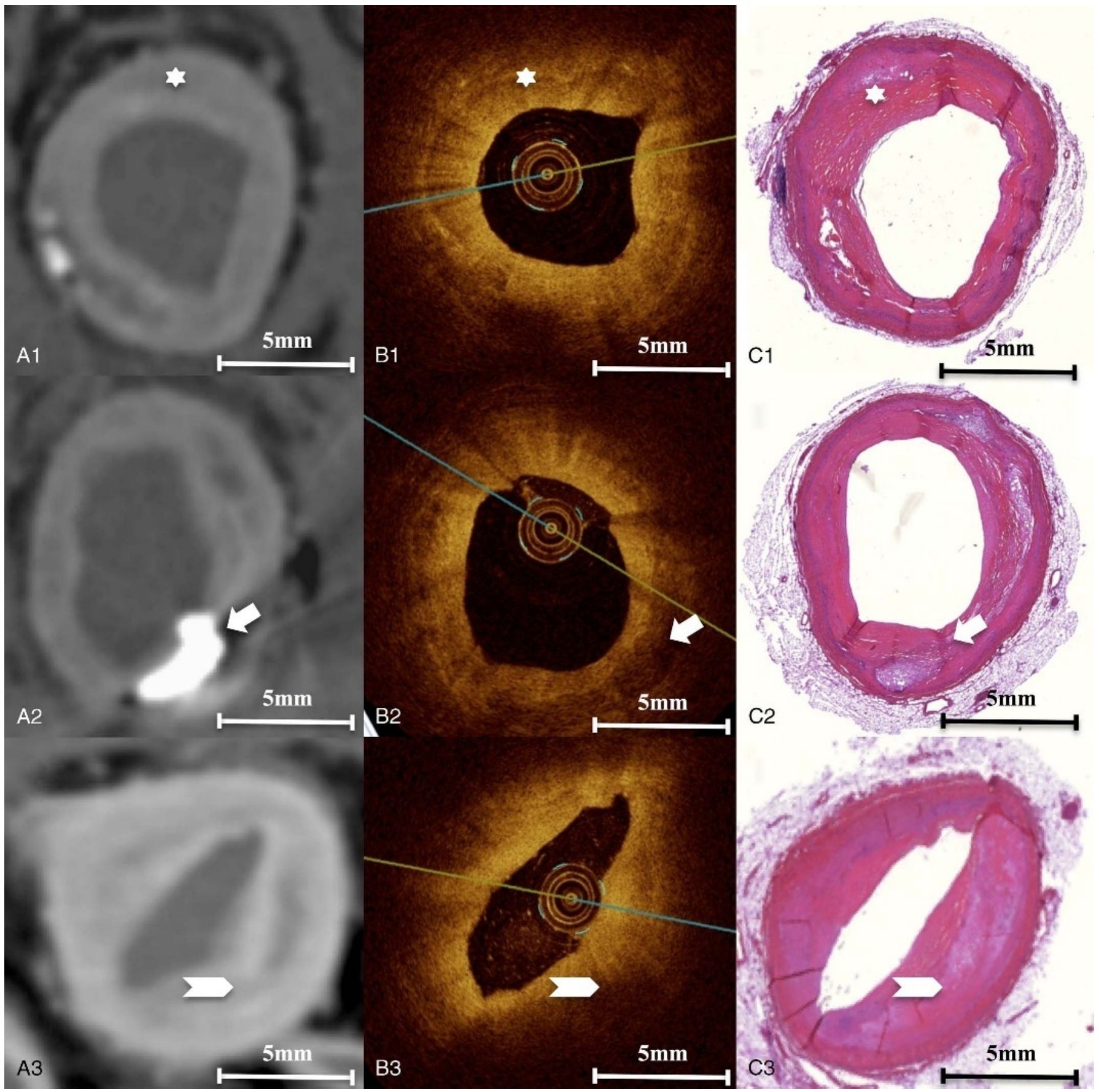

FIGURE 2. Atherosclerotic plaques of a right coronary artery (RCA) imaged by grating-based phase-contrast computed tomography (A1-A3) and corresponding cross sections from OCT measurements (B1-B3) and histopathology (hematoxylin-eosin staining) (C1-C3). Panels A1, B1, and C1 show a fibrous lesion; panels A2, B2, and C2 represent a calcified lesion, whereas panels A3, B3, and C3 show a lipid-rich lesion. Fibrous, calcified, and lipid-rich regions are marked with white asterisks, white arrows, and white bars, respectively. Figure 2 can be viewed online in color at www.investigativeradiology.com.

area may serve well to quantitatively assess coronary plaque burden. Optical coherence tomography is an established high-resolution imaging modality that serves as an in vivo criterion standard for plaque quantification and its use has greatly contributed to our current understanding of the natural history of coronary atherosclerosis. ${ }^{40}$ Previous studies could show the potential of OCT as a safe and effective modality for characterizing coronary atherosclerotic plaques, measuring and detecting cap thickness and visualizing microstructures near the lumen, for example, erosions or calcified nodules. ${ }^{41}$ Further studies are necessary to elucidate on the incremental role of OCT and gb-PCCT for advanced coronary plaque assessment.

It is interesting that gb-PCCT demonstrated high diagnostic accuracy for the detection and differentiation of plaque types, also beyond OCT. Although imperfect, gb-PCCT provided particularly higher diagnostic accuracy for the detection of LIP and CAL. One explanation is the superior penetration depth provided by gb-PCCT as compared with 


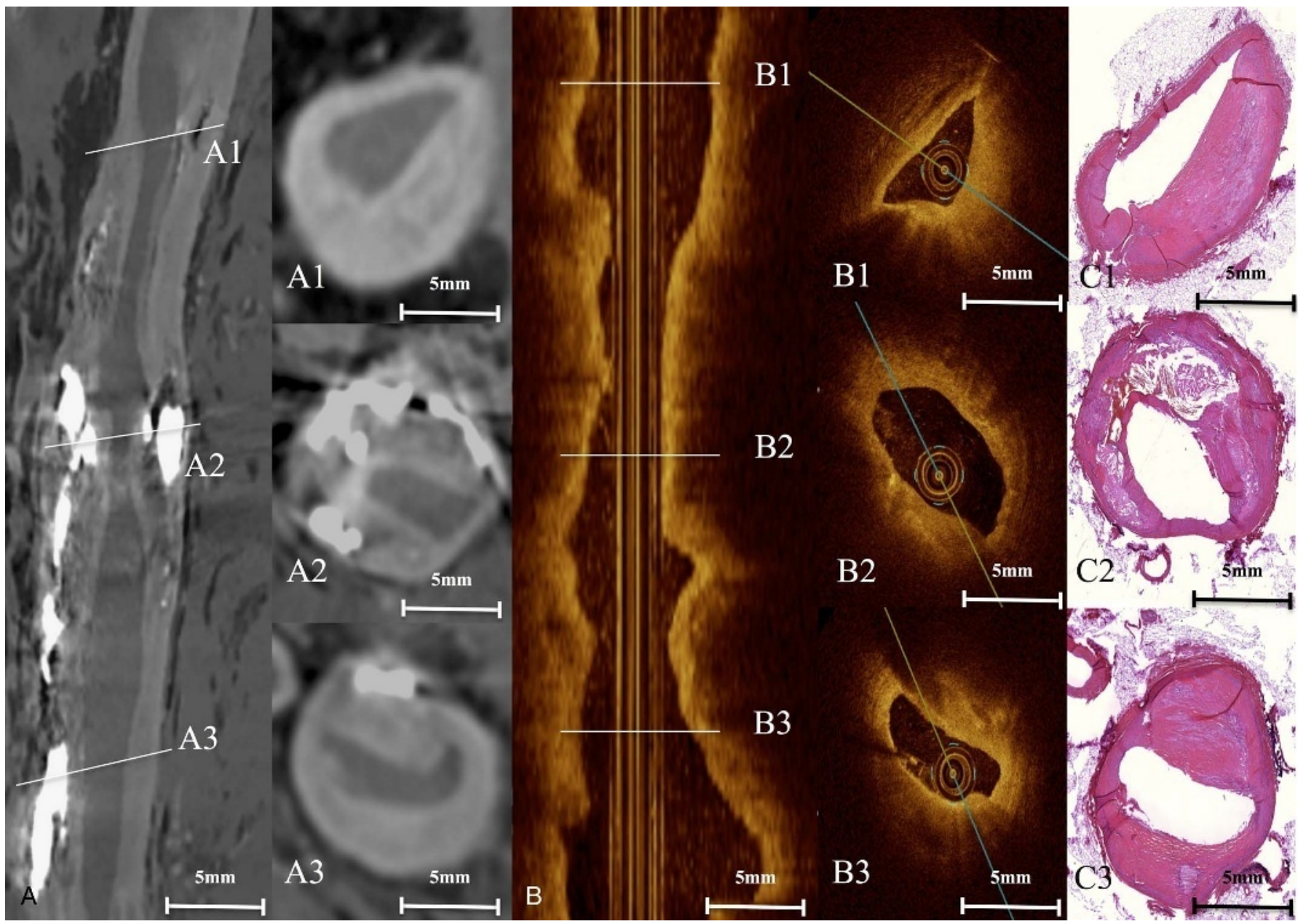

FIGURE 3. Longitudinal reconstruction of a left anterior descending artery (LAD) in grating-based phase-contrast computed tomography (gb-PCCT) (A) and optical coherence tomography (OCT) (B). Panels A1 to A3 show different cross sections in gb-PCCT of the LAD with a lipid-rich plaque (A1), a calcified plaque (A2), and a fibrous plaque (A3). Panels B1 to B3 show the corresponding sections in OCT. Panels C1 to C3 show the corresponding sections in histopathology (hematoxylin-eosin staining). In gb-PCCT, a 3D data set is acquired allowing for multiplanar reformatting and thus correct geometrical reconstruction of the vessel. OCT data does not contain complete 3D information; therefore, the longitudinal reconstruction of the vessel appears stretched and does not contain information about side branches. Figure 3 can be viewed online in color at www.investigativeradiology.com.

OCT, one of the well-known limitation of OCT. ${ }^{41}$ Furthermore, with a field of view (FOV) of $12 \mathrm{~cm}^{2}$, gb-PCCT provides images that cover the entire vessel cross section, whereas the ability of OCT to visualize vessel cross sections with an FOV of approximately $20 \mathrm{~mm}$ (scan diameter, $10 \mathrm{~mm}$ ) is limited. This aspect is highlighted in Figure 6, demonstrating the limited penetration depth of OCT in comparison to gb-PCCT particularly in large vessels. Moreover, the limited FOV and penetration depth of OCT resulted in a relatively high number of artifacts and the exclusion of multiple sections. Only few cross sections had to be excluded in gb-PCCT due to artifacts caused by small angle scattering at heavy calcifications, an example is shown in Supplement 2, Supplemental Digital Content 2, http://links.lww.com/RLI/A306. Thus, gb-PCCT may be advantageous for the assessment of large vessels, whereas both techniques provide similar feasibility in smaller vessels.

Notably, OCT is associated with a higher in-plane resolution, which may be advantageous in some applications. Our gb-PCCT
TABLE 1. Quantitative Measures of Lumen, Vessel, and Plaque Area by gb-PCCT in Comparison to Histopathology

\begin{tabular}{lccccc}
\hline Area, $\mathbf{~ m m}^{\mathbf{2}}$ & gb-PCCT & Histopathology & Difference & $\boldsymbol{P}$ & Pearson $\boldsymbol{r}$ \\
\hline Lumen & $5.5 \pm 4.1$ & $3.9 \pm 3.2$ & $1.6 \pm 0.9$ & $<0.0001$ & 0.85 \\
Vessel & $23.3 \pm 14.6$ & $14.3 \pm 9.3$ & $9.0 \pm 5.3$ & $<0.0001$ & 0.91 \\
Plaque & $17.9 \pm 11.5$ & $10.3 \pm 6.9$ & $7.6 \pm 4.6$ & $<0.0001$ & 0.87
\end{tabular}

gb-PCCT indicates grating-based phase-contrast computed tomography.
TABLE 2. Quantitative Measures of Lumen, Vessel, and Plaque Area by OCT in Comparison to Histopathology

\begin{tabular}{lrrrrc}
\hline Area, $\mathbf{~ m m}^{2}$ & \multicolumn{1}{c}{ OCT } & Histopathology & Difference & $\boldsymbol{P}$ & Pearson $\boldsymbol{r}$ \\
\hline Lumen & $4.6 \pm 3.0$ & $3.9 \pm 3.2$ & $0.7 \pm 0.2$ & $<0.0001$ & 0.64 \\
Vessel & $18.9 \pm 7.8$ & $14.3 \pm 9.3$ & $4.6 \pm 1.5$ & $<0.0001$ & 0.65 \\
Plaque & $14.3 \pm 5.5$ & $10.3 \pm 6.9$ & $4.0 \pm 1.4$ & $<0.0001$ & 0.61 \\
\hline
\end{tabular}

OCT indicates optical coherence tomography. 

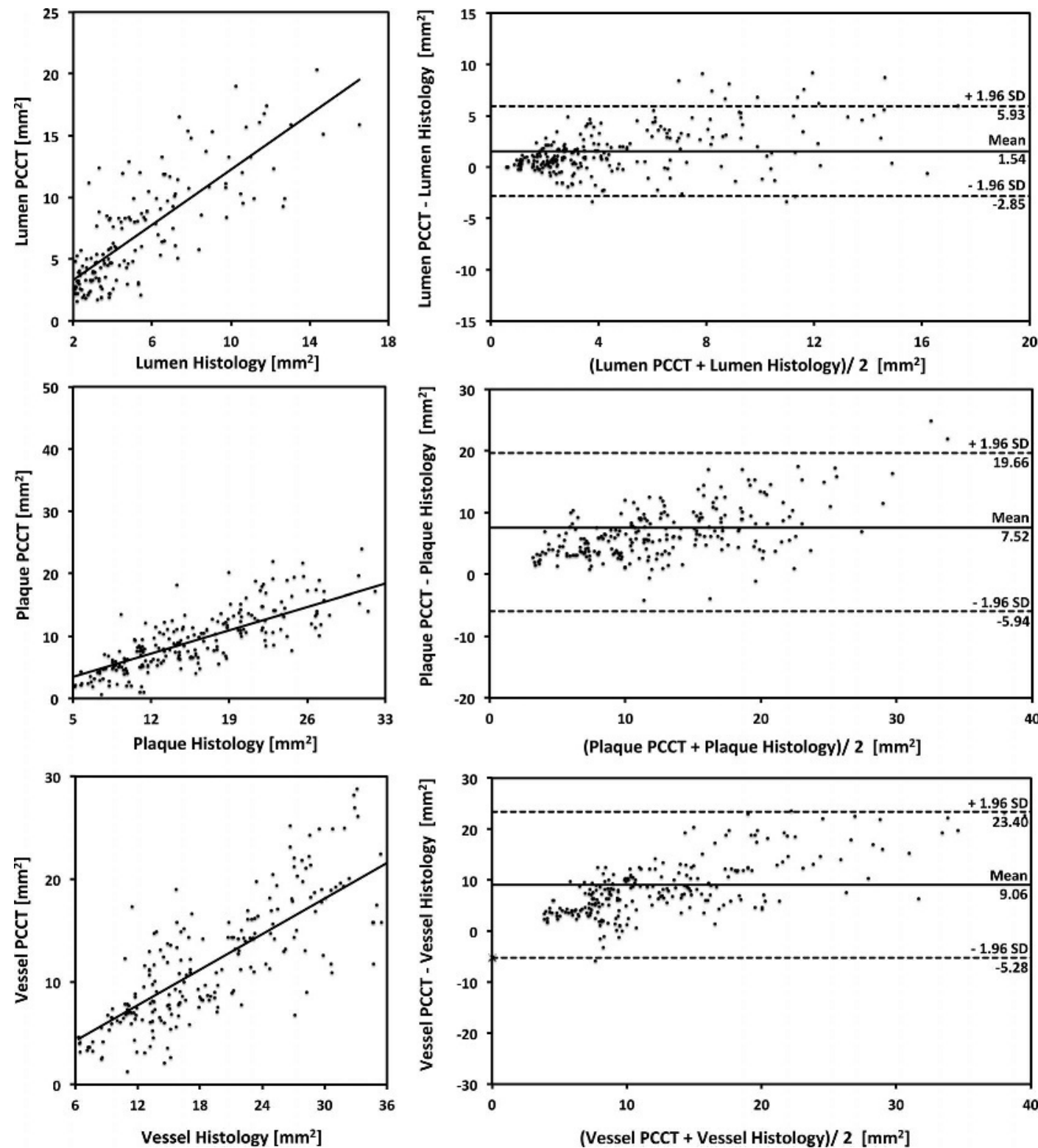

FIGURE 4. Scatter plots and Bland-Altman plots of quantitative measurements of lumen area, plaque area, and vessel area in grating-based phase-contrast computed tomography in comparison to histopathology measurements.

measurements were performed with an effective voxel size of $100^{3} \mu \mathrm{m}^{3}$ due to sample magnification of 1.72 in the setup, whereas the axial resolution of our OCT measurements was $15^{2} \mu \mathrm{m}^{2}{ }^{15}$ Therefore, OCT may be more useful for the characterization of smaller sized fibrous caps or macrophage imaging, which may be very relevant for distinct histopathological questions. ${ }^{42}$

It needs to be highlighted that gb-PCCT is an ex vivo imaging method with a setup not optimized for image acquisition time and radiation dose, which may certainly not be applicable to an in vivo imaging setting. The tube voltage of $40 \mathrm{kVp}$ is not sufficient for imaging tissues thicker than a few centimeters; thus for an in vivo imaging application in humans, further studies are needed. There are reports on successful translation of the gb-PCCT toward applications in humans; this includes developments toward, for example, the clinical dose compatible breast imaging ${ }^{32}$ or increasing FOV. ${ }^{43}$ Advances in novel monochromatic and compact $\mathrm{x}$-ray sources ${ }^{44}$ might help to overcome the current limitations of the gb-PCCT imaging and open completely new perspectives in a preclinical and clinical setting. Furthermore, gbPCCT is not only able to acquire phase and absorption contrast data simultaneously but also information on small angle scattering, the so-called dark field signal. Recently, the feasibility of the technique in an in vivo small-animal setting has been shown. ${ }^{45}$ This signal might be especially of interest in imaging microcalcifications, ${ }_{46,47}$ which have been associated with plaque instability. ${ }^{48,49}$ Given the aforementioned obstacles in acquiring the phase information signal in an in vivo setting, the dark field signal might be of special interest because it will potentially allow assessing microstructural changes much below the resolution of the imaging system. This issue has not been the focus of the present study but is subject to ongoing research. Advances in spectrum filtering, use of higher photon energies, applications of advanced stepping or Fourier-transform methods, increased pixel size, detectors with higher detection efficiency, and further measures to overcome these limitations are the focus of current research. 

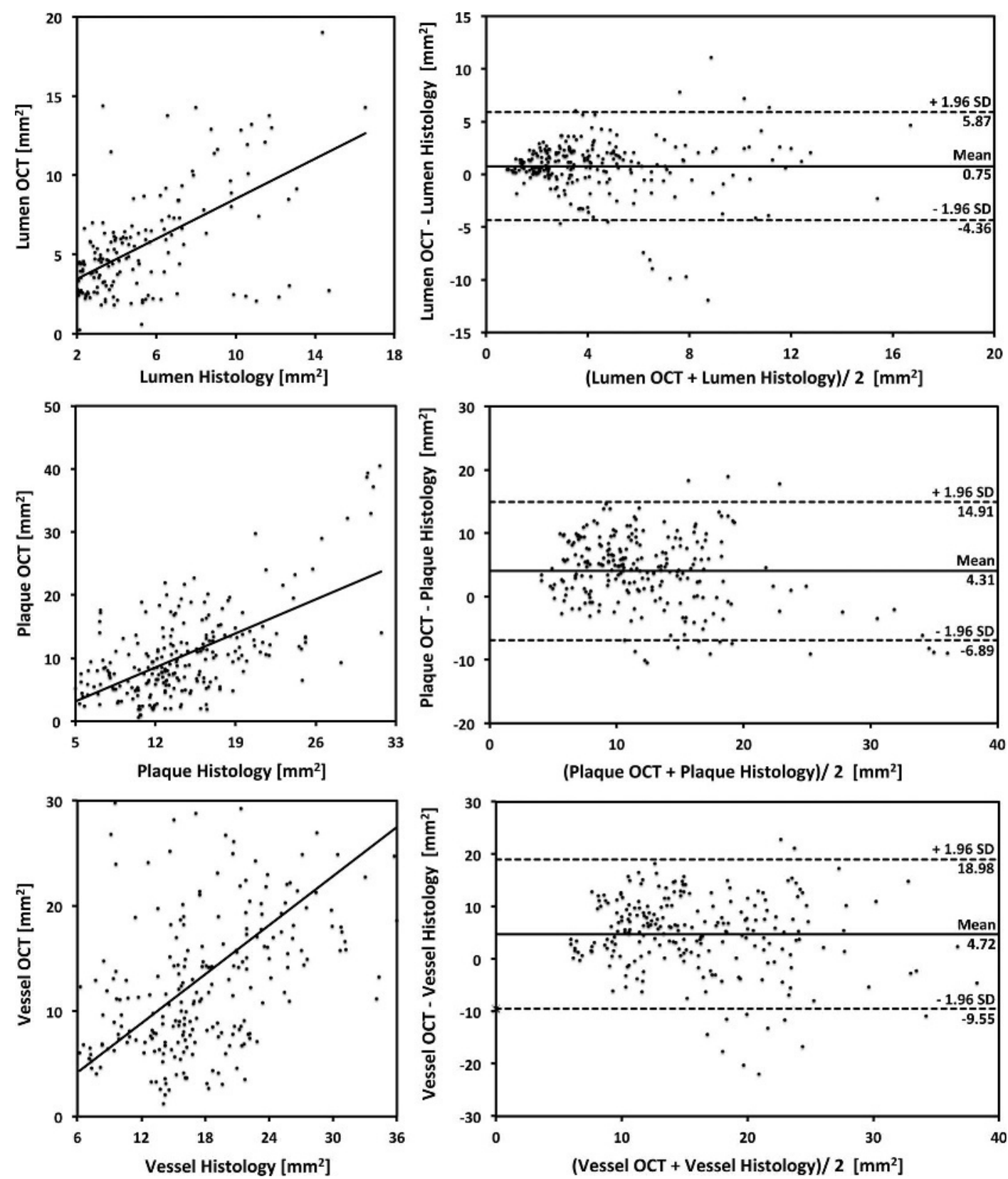

FIGURE 5. Scatter plots and Bland-Altman plots of quantitative measurements of lumen area, plaque area, and vessel area in optical coherence tomography in comparison to histopathology measurements.

Although the widespread use of gb-PCCT due to the necessary experimental setup is certainly limited at this point of time, there are several potential applications of this imaging method, including assessment of effects of novel pharmaceutical therapies ex vivo in animal models. ${ }^{50}$ Questions on the natural history relying on the 3D configuration of plaque may be particularly relevant for gb-PCCT.

TABLE 3. Diagnostic Accuracy of gb-PCCT

\begin{tabular}{lcccrrr}
\hline Plaque Component & Sensitivity (95\% CI) & Specificity (95\% CI) & PPV (95\% CI) & NPV (95\% CI) & \multicolumn{1}{c}{$\boldsymbol{\text { (95 }}$} \\
\hline Fibrous tissue & $0.98(0.96-0.99)$ & $1.00(0.92-1.00)$ & $1.00(0.98-1.00)$ & $0.94(0.88-0.98)$ & 0.96 & $<0.0001$ \\
Lipid-rich tissue & $0.95(0.89-0.98)$ & $0.95(0.90-0.97)$ & $0.88(0.81-0.94)$ & $0.98(0.94-0.99)$ & 0.89 & $<0.0001$ \\
Calcified tissue & $1.00(0.97-1.00)$ & $0.99(0.95-0.99)$ & $0.99(0.96-0.99)$ & $1.00(0.97-1.00)$ & 0.97 & $<0.0001$
\end{tabular}

gb-PCCT indicates grating-based phase-contrast computed tomography; CI, confidence interval; PPV, positive predictive value; NPV, negative predictive value. 
TABLE 4. Diagnostic Accuracy of OCT

\begin{tabular}{|c|c|c|c|c|c|c|}
\hline Plaque Component & Sensitivity (95\% CI) & Specificity (95\% CI) & PPV (95\% CI) & NPV (95\% CI) & $\kappa$ & $P$ \\
\hline Fibrous tissue & $1.00(0.97-1.00)$ & $0.66(0.35-0.88)$ & $0.98(0.93-0.98)$ & $1.00(0.59-1.00)$ & 0.81 & $<0.0001$ \\
\hline Lipid-rich tissue & $0.80(0.70-0.88)$ & $0.69(0.61-0.76)$ & $0.60(0.51-0.69)$ & $0.85(0.78-0.92)$ & 0.50 & $<0.0001$ \\
\hline Calcified tissue & $0.73(0.65-0.79)$ & $0.98(0.91-0.99)$ & $0.99(0.95-0.99)$ & $0.61(0.52-0.70)$ & 0.62 & $<0.0001$ \\
\hline
\end{tabular}

OCT indicates optical coherence tomography; CI, confidence interval; PPV, positive predictive value; NPV, negative predictive value.

However, further advances also with respect to standardization of image acquisition parameters are strongly warranted.

\section{Limitation}

Our results are derived from ex vivo formalin-fixated specimens, and thus, there may be differences to non-formalin-affected tissues. However, the study setup was not feasible to examine nonfixated samples, due to long acquisition times and the time intervals between preparation and scanning. A recent gb-PCCT-based study on fixated and nonfixated soft tissue suggested that fixation induces only minimal changes in quantitative tissue values. ${ }^{51}$ Misregistration of the different imaging modalities may have occurred; however, similar to previous research, coregistration relied on vessel landmarks, such as side branches. In addition, we included a relatively small number of samples, which limited our ability to perform more stratified subanalysis. Thus, our results provide some initial evidence on the feasibility of gb-PCCT for plaque assessment in comparison with OCT and are rather hypothesis generation. Clearly, confirmatory and more detailed research on specific questions is warranted.

\section{CONCLUSIONS}

In conclusion, gb-PCCT provides accurate assessment of coronary atherosclerotic plaque incremental to OCT ex vivo. Thus, the technique may be applied as a feasible, nondestructive modality for advanced plaque characterization in an experimental setting.
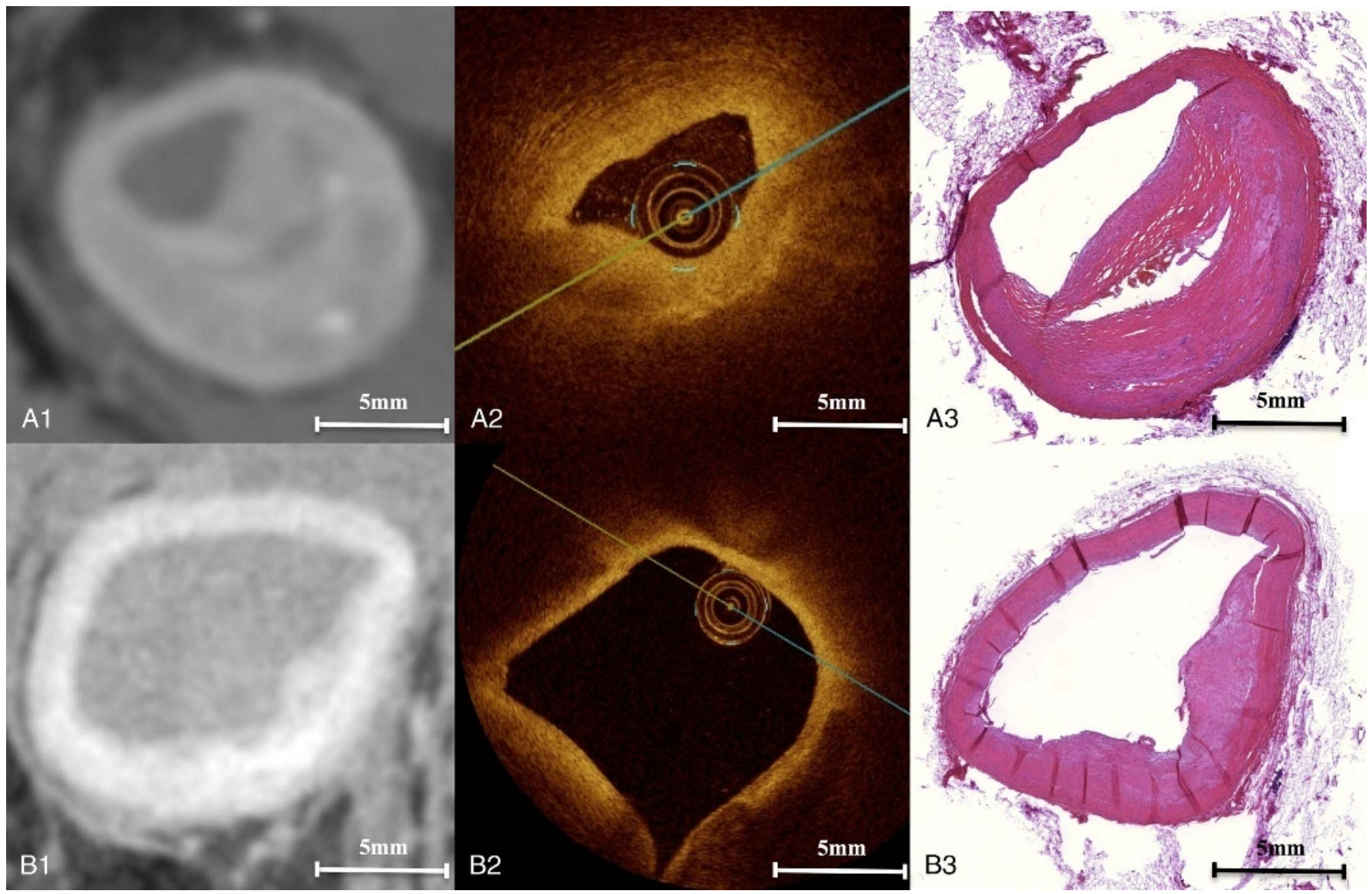

FIGURE 6. Stenosing atherosclerotic lesion (A1-A3), and a vessel with large diameter (B1-B3) in a left artery descending (LAD) in grating-based phase-contrast computed tomography (gb-PCCT) (A1 and B1), and the corresponding cross sections from OCT (A2 and B2) and histopathology (hematoxylin-eosin staining) (A3 and B3). These images demonstrate the low penetration depth of OCT in comparison to gb-PCCT and the limitations of OCT in vessels with large diameters (B1-B3). OCT is unable to depict the entire vessel wall measured (B2), whereas the gb-PCCT acquired data (A2) cover the entire vessel diameter. Figure 6 can be viewed online in color at www.investigativeradiology.com. 


\section{ACKNOWLEDGMENTS}

The authors acknowledge financial support through the European Research Council (ERC, H2020, AdG 695045), the DFG Cluster of Excellence Munich-Centre for Advanced Photonics (MAP), the DFG Gottfried Wilhelm Leibniz program, the Hans-und-Klementia-Langmatz-Foundation, and the support of the TUM Institute for Advanced Study, funded by the German Excellence Initiative. This work was carried out with the support of the Karlsruhe Nano Micro Facility (KNMF, www.kit.edu/knmf), a Helmholtz Research Infrastructure at Karlsruhe Institute of Technology (KIT). The study was funded by the German Research Foundation Cluster of Excellence Munich Center of Advanced Photonics (DFG EXC MAP) and the Hans-und-Klementia-Langmatz Foundation.

\section{REFERENCES}

1. Stary HC. Natural history and histological classification of atherosclerotic lesions: an update. Arterioscler Thromb Vasc Biol. 2000;20:1177-1178.

2. Hetterich H, Fill S, Herzen J, et al. Grating-based x-ray phase-contrast tomography of atherosclerotic plaque at high photon energies. Z Med Phys. 2013;23:194-203.

3. Hibi K, Kimura K, Umemura S. Clinical utility and significance of intravascular ultrasound and optical coherence tomography in guiding percutaneous coronary interventions. Circ J. 2015;79:24-33.

4. Schulman-Marcus J, Danad I, Truong QA. State-of-the-art updates on cardiac computed tomographic angiography for assessing coronary artery disease. Curr Treat Options Cardiovasc Med. 2015;17:398

5. Schuhbaeck A, Schmid J, Zimmer T, et al. Influence of the coronary calcium score on the ability to rule out coronary artery stenoses by coronary CT angiography in patients with suspected coronary artery disease. J Cardiovasc Comput Tomogr. 2016;10:343-350.

6. Hetterich H, Willner M, Habbel C, et al. X-ray phase-contrast computed tomography of human coronary arteries. Invest Radiol. 2015;50:686-694

7. Fitzgerald R. Phase-sensitive x-ray phase imaging. Physics Today. 2000;23:(6).

8. Donath T, Pfeiffer F, Bunk O, et al. Toward clinical X-ray phase-contrast CT: demonstration of enhanced soft-tissue contrast in human specimen. Invest Radiol. 2010;45:445-452.

9. Pfeiffer F, Kottler C, Bunk O, et al. Hard x-ray phase tomography with lowbrilliance sources. Phys Rev Lett. 2007;98:108105.

10. Shinohara M, Yamashita T, Tawa $\mathrm{H}$, et al. Atherosclerotic plaque imaging using phase-contrast x-ray computed tomography. Am J Physiol Heart Circ Physiol. 2008;294:H1094-H1100.

11. Pohle K, Achenbach S, Macneill B, et al. Characterization of non-calcified coronary atherosclerotic plaque by multi-detector row CT: comparison to IVUS. Atherosclerosis. 2007;190:174-180.

12. Weitkamp T, Diaz A, David C, et al. X-ray phase imaging with a grating interferometer. Opt Express. 2005;13:6296-6304.

13. Birnbacher L, Willner M, Velroyen A, et al. Experimental realisation of highsensitivity laboratory $\mathrm{x}$-ray grating-based phase-contrast computed tomography. Sci Rep. 2016;6:24022.

14. Pfeiffer F, Weitkamp T, Bunk O, et al. Phase retrieval and differential phasecontrast imaging with low-brilliance x-ray sources. Nat Phys. 2006:258-261.

15. St Jude Medical I. Therapy Guidance System. Ilumien Specs Sheet. 2011

16. Saam T, Herzen J, Hetterich H, et al. Translation of atherosclerotic plaque phasecontrast CT imaging from synchrotron radiation to a conventional lab-based x-ray source. PLoS One. 2013;8:e73513.

17. Hetterich H, Willner M, Fill S, et al. Phase-contrast CT: qualitative and quantitative evaluation of atherosclerotic carotid artery plaque. Radiology. 2014;271: $870-878$.

18. Einarsdottir H, Yaroshenko A, Velroyen A, et al. Computer-aided diagnosis of pulmonary diseases using x-ray darkfield radiography. Phys Med Biol. 2015;60: 9253-9268.

19. Hellbach K, Yaroshenko A, Meinel FG, et al. In vivo dark-field radiography for early diagnosis and staging of pulmonary emphysema. Invest Radiol. 2015;50: $430-435$.

20. Meinel FG, Schwab F, Yaroshenko A, et al. Lung tumors on multimodal radiographs derived from grating-based x-ray imaging-a feasibility study. Phys Med. 2014;30:352-357.

21. Meinel FG, Yaroshenko A, Hellbach K, et al. Improved diagnosis of pulmonary emphysema using in vivo dark-field radiography. Invest Radiol. 2014;49:653-658.

22. Velroyen A, Bech M, Tapfer A, et al. Ex vivo perfusion-simulation measurements of microbubbles as a scattering contrast agent for grating-based x-ray dark-field imaging. PLoS One. 2015; 10:e0129512.
23. Velroyen A, Yaroshenko A, Hahn D, et al. Grating-based x-ray dark-field computed tomography of living mice. EBioMedicine. 2015;2:1500-1506.

24. Yaroshenko A, Hellbach K, Yildirim AO, et al. Improved in vivo assessment of pulmonary fibrosis in mice using x-ray dark-field radiography. Sci Rep. 2015;5:17492.

25. Yaroshenko A, Meinel FG, Bech M, et al. Pulmonary emphysema diagnosis with a preclinical small-animal x-ray dark-field scatter-contrast scanner. Radiology. 2013;269:427-433.

26. Auweter SD, Herzen J, Willner M, et al. X-ray phase-contrast imaging of the breastadvances towards clinical implementation. Br J Radiol. 2014;87:20130606.

27. Grandl S, Scherer K, Sztrokay-Gaul A, et al. Improved visualization of breast cancer features in multifocal carcinoma using phase-contrast and dark-field mammography: an ex vivo study. Eur Radiol. 2015;25:3659-3668.

28. Grandl S, Willner M, Herzen J, et al. Evaluation of phase-contrast CT of breast tissue at conventional X-ray sources - presentation of selected findings. Z Med Phys 2013;23:212-221.

29. Grandl S, Willner M, Herzen J, et al. Visualizing typical features of breast fibroadenomas using phase-contrast CT: an ex-vivo study. PLoS One. 2014;9:e97101.

30. Gromann LB, Beque D, Scherer K, et al. Low-dose, phase-contrast mammography with high signal-to-noise ratio. Biomed Opt Express. 2016;7:381-391.

31. Scherer K, Birnbacher L, Chabior M, et al. Bi-directional X-ray phase-contrast mammography. PLoS One. 2014;9:e93502.

32. Scherer K, Willer K, Gromann L, et al. Toward clinically compatible phasecontrast mammography. PLoS One. 2015;10:e0130776.

33. Schleede S, Bech M, Grandl S, et al. X-ray phase-contrast tomosynthesis for improved breast tissue discrimination. Eur J Radiol. 2014;83:531-536.

34. Willner M, Herzen J, Grandl S, et al. Quantitative breast tissue characterization using grating-based x-ray phase-contrast imaging. Phys Med Biol. 2014;59: 1557-1571.

35. Hetterich $\mathrm{H}$, Webber $\mathrm{N}$, Willner $\mathrm{M}$, et al. AHA classification of coronary and carotid atherosclerotic plaques by grating-based phase-contrast computed tomography. Eur Radiol. 2016;26:3223-3233.

36. Winklhofer S, Peter S, Tischler V, et al. Diagnostic accuracy of quantitative and qualitative phase-contrast imaging for the ex vivo characterization of human coronary atherosclerotic plaques. Radiology. 2015;277:64-72.

37. Chamie D, Wang Z, Bezerra $\mathrm{H}$, et al. Optical coherence tomography and fibrous cap characterization. Curr Cardiovasc Imaging Rep. 2011;4:276-283.

38. Coletta J, Suzuki N, Nascimento BR, et al. Use of optical coherence tomography for accurate characterization of atherosclerosis [in English, Portuguese, Spanish] Arq Bras Cardiol. 2010;94:250-254, 268-272, 254-259.

39. Li J, Li X, Jing J, et al. Integrated intravascular optical coherence tomography (OCT) - ultrasound (US) catheter for characterization of atherosclerotic plaques in vivo. Conf Proc IEEE Eng Med Biol Soc. 2012;2012:3175-3178.

40. Sánchez-Elvira G, Coma-Canella I, Artaiz M, et al. Characterization of coronary plaques with combined use of intravascular ultrasound, virtual histology and optical coherence tomography. Heart Int. 2010;5:e12.

41. Jang IK, Tearney GJ, MacNeill B, et al. In vivo characterization of coronary atherosclerotic plaque by use of optical coherence tomography. Circulation. 2005 111:1551-1555.

42. Tearney GJ, Yabushita H, Houser SL, et al. Quantification of macrophage content in atherosclerotic plaques by optical coherence tomography. Circulation. 2003; 107:113-119.

43. Meiser J, Willner M, Schroter T, et al. Increasing the field of view in grating based X-ray phase contrast imaging using stitched gratings. J Xray Sci Technol. 2016;24: 379-388.

44. Eggl E, Dierolf M, Achterhold K, et al. The Munich Compact Light Source: initial performance measures. J Synchrotron Radiat. 2016;23:1137-1142.

45. Bech M, Tapfer A, Velroyen A, et al. In-vivo dark-field and phase-contrast X-ray imaging. Sci Rep. 2013;3:3209.

46. Wang Z, Hauser N, Singer G, et al. Non-invasive classification of microcalcification with phase-contrast X-ray mammography. Nat Commun. 2014;5:3797.

47. Scherer K, Braig E, Willer K, et al. Non-invasive differentiation of kidney stone types using X-ray dark-field radiography. Sci Rep. 2015;5:9527.

48. Vengrenyuk Y, Carlier S, Xanthos S, et al. A hypothesis for vulnerable plaque rupture due to stress-induced debonding around cellular microcalcifications in thin fibrous caps. Proc Natl Acad Sci US A. 2006;103:14678-14683.

49. Hutcheson JD, Maldonado N, Aikawa E. Small entities with large impact: microcalcifications and atherosclerotic plaque vulnerability. Curr Opin Lipidol. 2014;25:327-332.

50. Takeda M, Yamashita T, Shinohara M, et al. Beneficial effect of anti-platelet therapies on atherosclerotic lesion formation assessed by phase-contrast X-ray CT imaging. Int J Cardiovasc Imaging. 2012;28:1181-1191.

51. Willner M, Fior G, Marschner M, et al. Phase-contrast Hounsfield units of fixated and non-fixated soft-tissue samples. PLoS One. 2015;10:e137016. 